\title{
SC-KF Mobile Robot Localization: A Stochastic Cloning Kalman Filter for Processing Relative-State Measurements
}

\author{
Anastasios I. Mourikis, Student Member, IEEE, Stergios I. Roumeliotis, Member, IEEE, \\ and Joel W. Burdick, Member, IEEE
}

\begin{abstract}
This paper presents a new method to optimally combine motion measurements provided by proprioceptive sensors, with relative-state estimates inferred from feature-based matching. Two key challenges arise in such pose tracking problems: 1) the displacement estimates relate the state of the robot at two different time instants and 2) the same exteroceptive measurements are often used for computing consecutive displacement estimates, a process that renders the errors in these correlated. We present a novel stochastic cloning Kalman filtering (SC-KF) estimation algorithm that successfully addresses these challenges, while still allowing for efficient calculation of the filter gains and covariances. The proposed algorithm is not intended to compete with simultaneous localization and mapping (SLAM) approaches. Instead, it can be merged with any extended-Kalmanfilter-based SLAM algorithm to increase its precision. In this respect, the SC-KF provides a robust framework for leveraging additional motion information extracted from dense point features that most SLAM algorithms do not treat as landmarks. Extensive experimental and simulation results are presented to verify the validity of the proposed method and to demonstrate that its performance is superior to that of alternative position-tracking approaches.
\end{abstract}

Index Terms-Displacement estimates, relative-pose measurements, robot localization, state augmentation, stochastic cloning.

\section{INTRODUCTION}

A CCURATE localization is a prerequisite for a robot to meaningfully interact with its environment. The most commonly available sensors for acquiring localization information are proprioceptive sensors, such as wheel encoders, gyroscopes, and accelerometers, which provide information about the robot's motion. In dead reckoning (DR) [1], a robot's pose can be tracked from a starting point by integrating proprioceptive measurements over time. The limitation of DR is, however, that since no external reference signals are employed for correction, estimation errors accumulate over time, and the pose estimates

Manuscript received March 1, 2006; revised December 18, 2006. This paper was recommended for publication by Associate Editor W. Burgard and Editor L. Parker upon evaluation of the reviewers' comments. This work was supported in part by the University of Minnesota (DTC), in part by the NASA Mars Technology Program (MTP-1263201), and in part by the National Science Foundation (EIA-0324864, IIS-0643680).

A. I. Mourikis and S. I. Roumeliotis are with the Department of Computer Science and Engineering, University of Minnesota, Minneapolis, MN 55455 USA (e-mail: mourikis@cs.umn.edu; stergios@cs.umn.edu).

J. W. Burdick is with the Division of Engineering and Applied Science, California Institute of Technology, Pasadena, CA 91125 USA (e-mail: jwb@robotics.caltech.edu).

Digital Object Identifier 10.1109/TRO.2007.900610

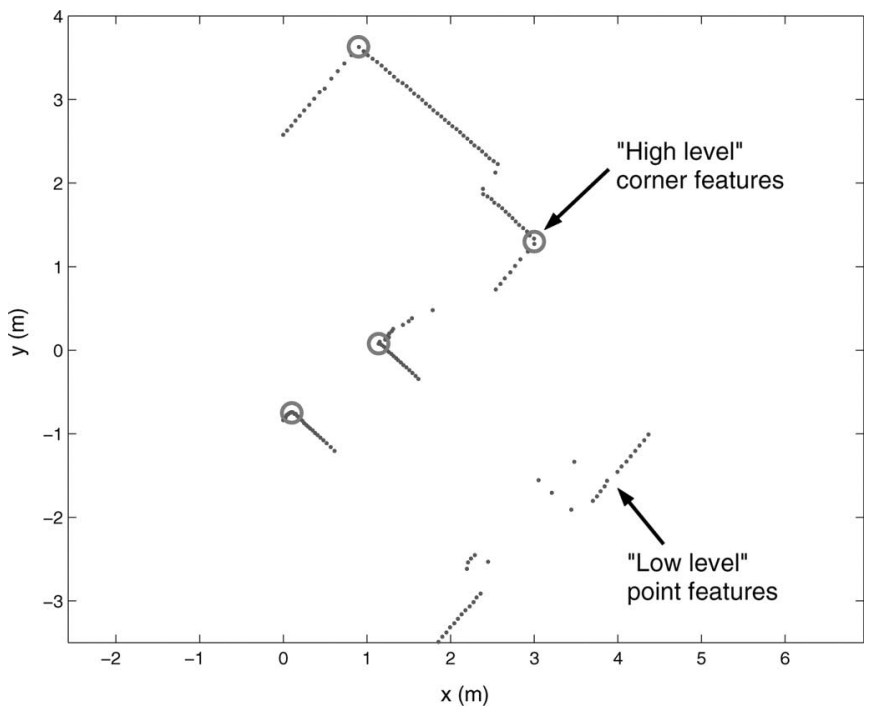

Fig. 1. Example of a planar laser scan and types of features observed. An algorithm has been employed to detect corners (intersections of line segments) in a laser scan. The extracted corner features can be used for performing SLAM, while all the remaining, "low-level" feature points, can be utilized in the SC-KF framework to improve the pose tracking accuracy.

drift from their real values. In order to improve localization accuracy, most algorithms fuse the proprioceptive measurements with data from exteroceptive sensors, such as cameras [2], [3] laser range finders [4], sonars [5], etc.

When an exteroceptive sensor provides information about the position of features with respect to the robot at two different time instants, it is possible (under necessary observability conditions) to create an inferred measurement of the robot's displacement. Examples of algorithms that process exteroceptive data to infer motion include laser scan matching [4], [6], [7], visionbased motion estimation techniques using stereoscopic [2], [3] and monocular [8] image sequences, and matching of sonar returns [5]. The inferred relative-state measurements that are derived from these can be integrated over time to provide pose estimates [3], or combined with proprioceptive sensory input in order to benefit from both available sources of positioning information [9], [10]. This paper focuses on how to optimally implement the latter approach using an extended Kalman filter (EKF) [11]. This paper does not consider the case in which the feature measurements are used for simultaneous localization and mapping (SLAM). However, as discussed in Section VI, our approach is complementary to SLAM, and can be employed to increase its accuracy (cf. Fig. 1). 
Two challenges arise when fusing proprioceptive and relativepose $^{1}$ measurements in an EKF. First, each displacement measurement relates the robot's state at two different time instants (i.e., the current time and previous time when exteroceptive measurements were recorded). However, the basic theory underlying the EKF requires that the measurements used for the state update be independent of any previous filter states. Thus, the "standard" formulation of the EKF, in which the filter's state comprises only the current state of the robot, is clearly not adequate for treating relative-state measurements.

A second challenge arises from the fact that when exteroceptive measurements are used to infer displacement, consecutive relative-state measurements will often be correlated. To understand the source of such correlations, consider, for example, the scenario in which a camera is employed to measure the pixel coordinates of the projections of the same landmarks at times $t_{k-1}, t_{k}$, and $t_{k+1}$. The errors in the measurements at time $t_{k}$ affect the displacement estimates for both time intervals $\left[t_{k-1}, t_{k}\right]$ and $\left[t_{k}, t_{k+1}\right]$, thereby rendering them correlated. Assuming that the measurements are uncorrelated (as is customarily done [2], [7], [10]) violates a basic assumption of EKF theory, leading to suboptimal or incorrect estimates for the robot's state and covariance. This fact has been generally overlooked in the literature, and to the best of our knowledge, no prior work exists that directly addresses this issue.

In this paper, we propose a direct approach to the problem of combining relative-pose measurements with proprioceptive measurements in order to improve the accuracy of DR. Our methodology augments the state vector of the Kalman filter to address the two aforementioned challenges. In particular, to properly account for the dependence on the robot's state estimates at different time instants, we augment the Kalman filter state to include two instances (or clones) of the state estimatehence the name stochastic cloning Kalman filter (SC-KF) [9]. Moreover, in order to appropriately treat the correlations between consecutive displacement estimates, we further augment the state to include the most recent exteroceptive measurements [11]. With these state augmentations, the displacement measurements can be expressed as functions of the current filter state, and thus, an EKF framework can be employed.

The following section reviews existing approaches for processing relative-state measurements, while Section III presents the structure of the correlations between consecutive measurements, and investigates their effect on displacement-only propagation of the robot state. Section IV describes in detail the $\mathrm{SC}-\mathrm{KF}$ algorithm. Section V presents extensions of the SC-KF methodology, while Section VI discusses its relation to SLAM. In Section VII, it is shown that the attained position-tracking accuracy is superior to that of existing approaches. Finally, the conclusions are presented in Section VIII.

1 Throughout this paper, the terms "displacement measurement" and "relative-pose measurement" are used interchangeably to describe a measurement of the robot's motion that is inferred from exteroceptive measurements Depending on the type and number of the available features, either all or a subset of the degrees of freedom of motion may be determined (cf. Section VII-A.2).

\section{RELATED APPROACHES}

Displacement measurements can be treated as average velocity measurements during the corresponding time interval. These average velocities can then be combined with velocity measurements obtained from the robot's proprioceptive sensors to improve their accuracy. However, this approach is applicable only if the relative-state measurements are made at a rate equal to or higher than that of the proprioceptive sensors, which is rarely the case in practice. Alternatively, the robot's velocity estimate could be included in the state vector, and the average velocity estimates could then be used as instantaneous velocity pseudomeasurements in the EKF update step [12]. The shortcoming of this method is that treating an average velocity measurement as an instantaneous one can introduce significant errors when the rate of the displacement measurements is low. A different solution, proposed in [10], is to use the previous robot position estimates for converting the relative pose measurements to absolute position pseudomeasurements. However, since these pseudoabsolute measurements are correlated with the state, their covariance matrix has to be artificially inflated to guarantee consistency, thus resulting in suboptimal estimation (cf. Section VII-A).

Contrary to the preceding ad hoc methods for processing displacement measurements, several existing approaches employ these measurements to impose constraints between consecutive robot poses. Algorithms that only use displacement measurements for propagating the robot's state estimate are often described as sensor-based odometry methods [2], [4]. In these algorithms, only the last two robot poses (the current and previous one) are ever considered. While our stochastic cloning approach (which was first introduced in [9]) also relies only upon the last two robot poses, tracking is achieved by fusing the displacement measurements with proprioceptive information. Therefore, our method can be seen as an "enhanced" form of odometry. On the other hand, several existing approaches maintain a state vector comprising a history of robot poses, and use the displacement measurements to impose constraints between pairs of these poses. In [13], the robot's orientation errors are assumed to be temporally uncorrelated, which transforms the problem of optimizing the network of robot poses into a linear one, where only the robot positions are estimated. In [14]-[16], the full 3-D robot pose of an autonomous underwater vehicle is estimated, while in [7] and [17], displacement constraints are employed for estimating the pose history of a robot in two dimensions.

All of the approaches discussed so far do not properly account for the correlations that exist between consecutive displacement estimates, as they are assumed to be independent. However, as shown in Section III, this assumption does not generally hold. One could avoid such correlations by using each feature measurement in the computation of only one displacement estimate [14]. For example, half the measurements at each time step can be used to estimate the previous displacement, and the other half to estimate the next one. The drawback of this methodology is that incorporating only part of the available exteroceptive measurements when computing each relative-pose estimate results in less accurate displacement estimates. In our paper, all available measurements are used to compute the relative-pose 
measurements at every time step, and the correlations introduced by this process are explicitly identified and accounted for.

Solutions to the well-known SLAM problem (cf. Section VI) "circumvent" the problem of treating the displacement measurements by including the features' positions in the state vector, and jointly estimating the robot's and features' state. While SLAM offers high localization accuracy, the computational complexity associated with the estimation of the positions of a large number of features may be prohibitive for some real-time applications (e.g., autonomous aircraft landing). Thus, there exists a need for methods that enable direct processing of the displacement measurements at a lower computational cost.

In this paper, we propose an algorithm for optimally fusing the potentially correlated relative displacement estimates with proprioceptive measurements. The SC-KF considers exteroceptive measurements in pairs of consecutive measurements that are first processed to create an inferred relative-pose measurement, and then, fused with the proprioceptive measurements. The sole objective of the SC-KF is to estimate the robot's state, and therefore, the states of features used for deriving the displacement measurements are not estimated. Hence, the proposed algorithm can optimally fuse relative-pose measurements with the minimum computational overhead (Section IV-D). The proposed method can be used either as a stand-alone localization algorithm, or combined with SLAM in order to increase its localization accuracy (cf. Section VI).

\section{Relative-Pose MeAsurement CoRrelations}

Before presenting the SC-KF algorithm, we first study the structure of the correlations between consecutive displacement estimates. Let $z_{k}$ and $z_{k+m}$ denote the vectors of exteroceptive measurements at times $t_{k}$ and $t_{k+m}$, respectively, whose noise covariance matrices are $R_{k}$ and $R_{k+m}$. These are measurements, for example, of range and bearing from a laser range finder, or of bearing from a camera. By processing these measurements (e.g., via laser scan matching), an estimate $z_{k, k+m}$ for the change in the robot pose between times $t_{k}$ and $t_{k+m}$ is computed as a function (either closed form or implicit) of $z_{k}$ and $z_{k+m}$ :

$$
z_{k, k+m}=\xi\left(z_{k}, z_{k+m}\right) .
$$

Linearization of (1) relates the error in the displacement estimate, $\widetilde{z}_{k, k+m}$, to errors in the exteroceptive measurements ${ }^{2}$ :

$$
\widetilde{z}_{k, k+m} \simeq J_{k, k+m}^{k} \widetilde{z}_{k}+J_{k, k+m}^{k+m} \widetilde{z}_{k+m}+n_{k, k+m}
$$

where the noise term $n_{k, k+m}$ arises from inaccuracies in the displacement-estimation algorithm (e.g., errors due to feature matching [6]). We assume that the exteroceptive measurement errors $\widetilde{z}_{k}$ and $\widetilde{z}_{k+m}$ and the noise term $n_{k, k+m}$ are zero-mean and independent, an assumption that holds in most practical cases if proper sensor characterization is performed. In (2), $J_{k, k+m}^{k}$ and $J_{k, k+m}^{k+m}$ are the Jacobians of the function $\xi$ with

\footnotetext{
${ }^{2}$ The "hat" symbol $\widehat{ }$ is used to denote the estimated value of a quantity, while the "tilde" symbol $\sim$ is used to signify the error between the actual value of a quantity and its estimate. The relationship between a variable $x$, its estimate $\widehat{x}$, and the error $\widetilde{x}$ is $\widetilde{x}=x-\widehat{x}$.
}

respect to $z_{k}$ and $z_{k+m}$, respectively, i.e.,

$$
J_{k, k+m}^{k}=\nabla_{z_{k}} \xi \quad \text { and } \quad J_{k, k+m}^{k+m}=\nabla_{z_{k+m}} \xi
$$

Generally, not all feature measurements in the vector $z_{k}$ are used to estimate displacement. For example, in laser scan matching, there usually exists only partial overlap between consecutive scans, and therefore, not all laser returns are matched. As a result, if $M_{k}$ denotes the number of feature measurements in $z_{k}^{T}=\left[\left(z_{k}\right)_{1}^{T} \cdots\left(z_{k}\right)_{M_{k}}^{T}\right]$, the $i$ th component of the Jacobian matrices $J_{k, k+m}^{k}$ and $J_{k, k+m}^{k+m}$ takes the form

$$
\begin{aligned}
& \left(J_{k, k+m}^{t}\right)_{i} \\
& \quad= \begin{cases}\nabla_{\left(z_{t}\right)_{i}} \xi, & i \text { th feature used to compute } z_{k, k+m} \\
0, & \text { else }\end{cases}
\end{aligned}
$$

for $i=1, \ldots, M_{k}$, and $t=k, k+m$. Thus, for some applications, the Jacobians may be significantly sparse.

Our goal is to compute the correlation between the displacement estimates for the time intervals $\left[t_{k-\ell}, t_{k}\right]$ and $\left[t_{k}, t_{k+m}\right]$, which is defined as $E\left\{\widetilde{z}_{k-\ell, k} \widetilde{z}_{k, k+m}^{T}\right\}$. For this purpose, we employ (2) and the independence of exteroceptive measurement errors at different time steps to obtain

$$
\begin{aligned}
E\left\{\widetilde{z}_{k-\ell, k} \widetilde{z}_{k, k+m}^{T}\right\} & =J_{k-\ell, k}^{k} E\left\{\widetilde{z}_{k} \widetilde{z}_{k}^{T}\right\} J_{k, k+m}^{k T} \\
& =J_{k-\ell, k}^{k} R_{k} J_{k, k+m}^{k T} .
\end{aligned}
$$

Note that exteroceptive measurements typically consist of observations of a number of features detected in the robot's vicinity (e.g., distance and bearing to points on a wall or the image coordinates of visual features). In such cases, the measurements of the individual features are mutually independent, and therefore, the covariance matrix $R_{k}$ is block diagonal. In light of (3), when $R_{k}$ is block diagonal, expression (4) is equal to zero only if different features are used to estimate displacement in consecutive time intervals (i.e., if nonoverlapping subsets of $z_{k}$ are matched with $z_{k-\ell}$ and $z_{k+m}$, respectively). Clearly, this is not the case in general, and thus, consecutive displacement estimates are, in most cases, not independent.

\section{A. State Propagation Based Exclusively on Displacement Measurements}

We now show how the preceding analysis can be employed in the simple setting where the robot state estimates are propagated using displacement measurements only. This is an important special case, which has been extensively studied in the literature (examples include visual odometry [2], [3], laser-based odometry [4], etc.). Once the displacement estimate between $t_{k}$ and $t_{k+1}$ has been computed [cf. (1)], an estimate for the robot's pose at $t_{k+1}$ is obtained by combining the previous pose estimate and the displacement measurement:

$$
\widehat{X}_{k+1}=g\left(\widehat{X}_{k}, z_{k, k+1}\right) .
$$

By linearizing this equation, the pose errors at $t_{k+1}$ can be related to the errors in the previous state estimate and displacement measurement:

$$
\widetilde{X}_{k+1} \simeq \Phi_{k} \widetilde{X}_{k}+\Gamma_{k} \widetilde{z}_{k, k+1}
$$

where $\Phi_{k}$ and $\Gamma_{k}$ represent the Jacobians of the state propagation function $g\left(\widehat{X}_{k}, z_{k, k+1}\right)$ with respect to the previous pose and the 
relative pose measurement, respectively:

$$
\Phi_{k}=\nabla_{\widehat{X}_{k}} g, \quad \Gamma_{k}=\nabla_{z_{k, k+1}} g .
$$

The covariance matrix of the pose estimates is propagated by

$$
\begin{aligned}
P_{k+1}= & E\left\{\widetilde{X}_{k+1} \widetilde{X}_{k+1}^{T}\right\} \\
= & \Phi_{k} P_{k} \Phi_{k}^{T}+\Gamma_{k} R_{k, k+1} \Gamma_{k}^{T} \\
& +\Phi_{k} E\left\{\widetilde{X}_{k} \widetilde{z}_{k, k+1}^{T}\right\} \Gamma_{k}^{T}+\Gamma_{k} E\left\{\widetilde{z}_{k, k+1} \widetilde{X}_{k}^{T}\right\} \Phi_{k}^{T}
\end{aligned}
$$

where $R_{k, k+1}$ denotes the noise covariance of the displacement estimates. A common simplifying assumption in the literature (e.g., [2], [7]) is that the measurement noise $\widetilde{z}_{k, k+1}$ and state error $\widetilde{X}_{k}$ are uncorrelated, and thus, the last two terms in (8) are set to zero. However, this assumption, generally, does not hold when correlations exist between consecutive displacement estimates. In particular, by linearizing the state propagation equation at $t_{k}$, we obtain [cf. (6)]

$$
\begin{aligned}
& E \\
& \left.\qquad \widetilde{z}_{k, k+1} \widetilde{X}_{k}^{T}\right\}=E\left\{\widetilde{z}_{k, k+1}\left(\Phi_{k-1} \widetilde{X}_{k-1}+\Gamma_{k-1} \widetilde{z}_{k-1, k}\right)^{T}\right\} \\
& =E\left\{\widetilde{z}_{k, k+1} \widetilde{X}_{k-1}^{T}\right\} \Phi_{k-1}^{T}+E\left\{\widetilde{z}_{k, k+1} \widetilde{z}_{k-1, k}^{T}\right\} \Gamma_{k-1}^{T} \\
& =E\left\{\widetilde{z}_{k, k+1} \widetilde{z}_{k-1, k}^{T}\right\} \Gamma_{k-1}^{T} .
\end{aligned}
$$

Note that the error term $\widetilde{X}_{k-1}$ depends on the measurement errors of all exteroceptive measurements up to and including time $t_{k-1}$, while the error term $\widetilde{z}_{k, k+1}$ depends on the measurement errors at times $t_{k}$ and $t_{k+1}$ [cf. (2)]. As a result, the errors $\widetilde{X}_{k-1}$ and $\widetilde{z}_{k, k+1}$ are independent. Therefore, by applying the zero-mean assumption for the error $\widetilde{z}_{k, k+1}$, we obtain $E\left\{\widetilde{z}_{k, k+1} \widetilde{X}_{k-1}^{T}\right\}=0$. By employing the result of (4) and substituting (9) in (8), we obtain the following expression for the propagation of the pose covariance in the case of inferred displacement measurements:

$$
\begin{aligned}
P_{k+1}= & \Phi_{k} P_{k} \Phi_{k}^{T}+\Gamma_{k} R_{k, k+1} \Gamma_{k}^{T} \\
& +\Phi_{k} \Gamma_{k-1} J_{k-1, k}^{k} R_{k} J_{k, k+1}^{k T} \Gamma_{k}^{T} \\
& +\Gamma_{k} J_{k, k+1}^{k} R_{k} J_{k-1, k}^{k T} \Gamma_{k-1}^{T} \Phi_{k}^{T} .
\end{aligned}
$$

In Algorithm 1, the steps necessary for propagating the robot's state estimate and its covariance using displacement measurements are outlined.

\footnotetext{
Algorithm 1 Pose Estimation Based on Relative-Pose Measurements

Initialization:

- Initialize the robot covariance matrix when the first exteroceptive measurement is received

Propagation: For each exteroceptive measurement:

- compute the displacement measurement using (1) and its Jacobians with respect to the current and previous exteroceptive measurement using (3).

- propagate the robot state estimate using (5)

- compute the Jacobians of the pose propagation function using (7)

- propagate the robot pose covariance matrix via (10) (during the first iteration, use only the first two terms)

- compute and store the matrix product $\Gamma_{k} J_{k, k+1}^{k+1}$ that will be used in the next iteration
}

\section{B. Investigation of the Effects of Correlations}

Based on numerous experiments and simulation tests, we have observed that when the correlations between displacement measurements are accounted for, the covariance estimate is typically smaller than when the correlations are ignored. We attribute this result to the fact that the correlation between consecutive relative-pose estimates tends to be negative. An intuitive explanation for this observation can be given by means of a simple example for 1-D motion. Consider a robot moving on a straight line and recording measurements $z_{k}$ of the distance to a single feature on the same line. If at time $t_{k}$, the error in the distance measurement is equal to $\epsilon_{k}>0$, this error will contribute toward underestimating the robot's displacement during the interval $\left[t_{k-1}, t_{k}\right]$, but will contribute toward overestimating the displacement during the interval $\left[t_{k}, t_{k+1}\right]$. Therefore, the error $\epsilon_{k}$ has opposite effects on the two displacement estimates, rendering them negatively correlated.

In this 1-D example, it is interesting to examine the time evolution of the covariance when the correlations are properly treated. Note that the robot's displacement can be computed as the difference of two consecutive distance measurements, i.e., $z_{k, k+1}=z_{k}-z_{k+1}$. If the covariance of the individual distance measurements is equal to $R_{k}=R_{k+1}=\sigma^{2}$, then the covariance of $z_{k, k+1}$ is equal to $R_{k, k+1}=2 \sigma^{2}$. Moreover, for this example, it is easy to see that all the Jacobians in (10) are constant, and given by $J_{k, k+1}^{k}=1, J_{k-1, k}^{k}=-1, \Phi_{k}=\Gamma_{k}=\Gamma_{k-1}=1$. Substituting these values in (10), we obtain the following equation for covariance propagation in this case:

$$
P_{k+1}=P_{k}+R_{k, k+1}-R_{k}-R_{k}=P_{k} \text {. }
$$

We, thus, see that the covariance of the robot's position estimate remains constant during propagation when the correlations are properly treated. This occurs because the error in the measurement $z_{k}$ effectively "cancels out." On the other hand, if the correlations between consecutive displacement measurements are ignored, we obtain

$$
P_{k+1}=P_{k}+R_{k, k+1}=P_{k}+2 \sigma^{2} .
$$

In this case, the position covariance increases linearly, a result that does not reflect the evolution of the true state uncertainty.

In the context of this 1-D example, we next study the time evolution of the covariance when features come in and out of the robot's field of view (FOV). Assume that a uniform distribution of features, with density $\rho$, exists on the line, and that the robot's FOV is limited to $\ell_{\max } / 2$ in each direction. If the robot moves by $\Delta \ell$ between the time instants the measurements are recorded, then the overlap in the FOV at consecutive time instants is $\ell_{\max }-\Delta \ell$. Within this region lie $M_{k}=\rho\left(\ell_{\max }-\right.$ $\Delta \ell)$ features, whose measurements are used for displacement estimation. The least squares displacement estimate is given by

$$
z_{k, k+1}=\frac{1}{M_{k}} \sum_{i=1}^{M_{k}}\left(\left(z_{k}\right)_{i}-\left(z_{k+1}\right)_{i}\right)
$$

where $\left(z_{k}\right)_{i}$ and $\left(z_{k+1}\right)_{i}$ are the measurements to the $i$ th feature at times $t_{k}$ and $t_{k+1}$, respectively. The covariance of $z_{k, k+1}$ is 
given by

$$
R_{k, k+1}=\frac{2 \sigma^{2}}{M_{k}}=\frac{2 \sigma^{2}}{\rho\left(\ell_{\max }-\Delta \ell\right)}
$$

Thus, if one ignores the correlations between consecutive displacement estimates, the covariance propagation equation is

$$
P_{k+1}^{\mathrm{NC}}=P_{k}^{\mathrm{NC}}+\frac{2 \sigma^{2}}{\rho\left(\ell_{\max }-\Delta \ell\right)}
$$

where the superscript NC denotes the fact that no correlations are treated. At the end of a path of length $\ell_{\text {total }}$ (i.e., after $\ell_{\text {total }} / \Delta \ell$ propagation steps), the estimated covariance of the robot position, starting from a zero initial value, will be given by

$$
P_{\text {final }}^{\mathrm{NC}}=\frac{\ell_{\text {total }}}{\Delta \ell} \frac{2 \sigma^{2}}{\rho\left(\ell_{\max }-\Delta \ell\right)} .
$$

We now derive the corresponding covariance equations for the case that the correlations are properly incorporated. Since the robot moves by a distance $\Delta \ell$ between the time instants when the measurements are recorded, the number of features that are observed at three consecutive time instants (i.e., $t_{k-1}, t_{k}$, and $\left.t_{k+1}\right)$ is $\rho\left(\ell_{\max }-2 \Delta \ell\right)$. Employing this observation to evaluate the Jacobians in (10) yields the following expression for the propagation of the covariance:

$$
P_{k+1}=P_{k}+\frac{2 \sigma^{2} \Delta \ell}{\rho\left(\ell_{\max }-\Delta \ell\right)^{2}}, \text { for } \ell_{\max }>2 \Delta \ell .
$$

Note that if $\ell_{\max }<2 \Delta \ell$, no overlap exists between the FOV at times $t_{k-1}$ and $t_{k+1}$, and thus, no feature measurement is used twice for computing displacement estimates. In that case, expression (15) is exact. At the end of a path of length $\ell_{\text {total }}$, the covariance of the robot position is

$$
P_{\text {final }}=\frac{2 \sigma^{2} \ell_{\text {total }}}{\rho\left(\ell_{\max }-\Delta \ell\right)^{2}}, \text { for } \ell_{\max }>2 \Delta \ell .
$$

From (16) and (18), we see that for $\ell_{\max }>2 \Delta \ell$, the following relation holds:

$$
\frac{P_{\text {final }}^{\mathrm{NC}}}{P_{\text {final }}}=\frac{\ell_{\max }-\Delta \ell}{\Delta \ell}>1 .
$$

This shows that when the correlations are ignored, the resulting covariance estimates are larger, similarly to what is observed in the experimental results.

Fig. 2 plots the variance in the robot's position at the end of a trajectory of length $\ell_{\text {total }}=100 \mathrm{~m}$, as a function of the size of the robot's displacement between consecutive measurements. The solid line corresponds to the case when the correlations between displacement measurements are accounted for [cf. (18)], while the dashed line corresponds to the case when these are ignored [cf. (16)]. The parameters used to generate this plot are: feature density $\rho=5$ features $/ \mathrm{m}$, robot FOV $\ell_{\max }=$ $10 \mathrm{~m}$, and standard deviation of each distance measurement $\sigma=0.2 \mathrm{~m}$. It is important to note that when the correlations between consecutive measurements are accounted for, the final uncertainty is a monotonically increasing function of the displacement between measurements $\Delta \ell$. This agrees with intuition, which dictates that when measurements occur less frequently, the accuracy of the final state estimates deteriorates.

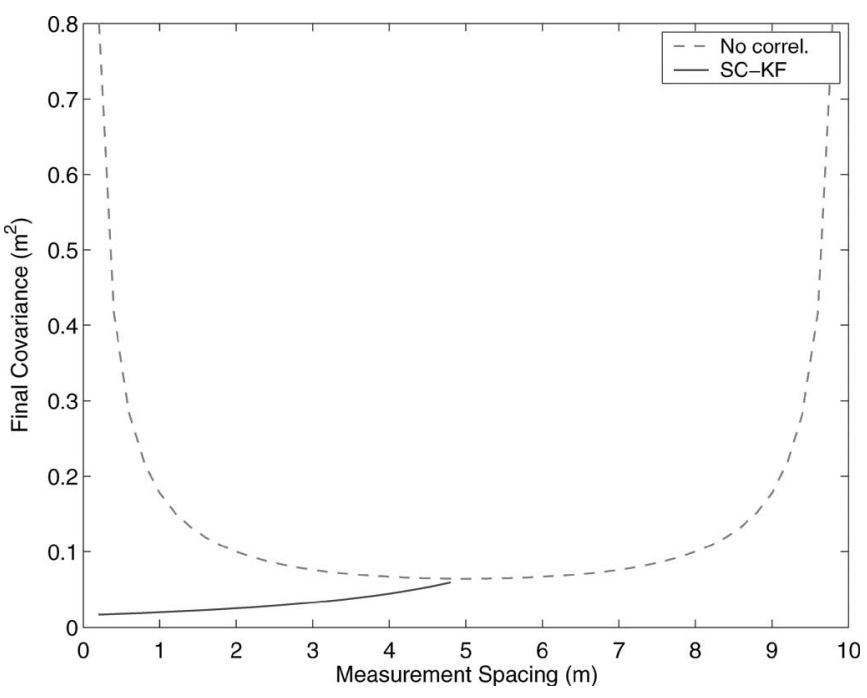

Fig. 2. Covariance estimates at the end of a 100-m trajectory using the expression of (10) (solid line) versus when the correlations between consecutive displacement measurements are not accounted for (dashed line). Note that when the measurements occur more than $5 \mathrm{~m}$ apart, no correlations exist, and the two estimates are identical.

However, when the correlations between displacement measurements are ignored, the covariance estimates do not have this property. Fig. 2 shows that for $\Delta \ell<\ell_{\max } / 2=5 \mathrm{~m}$, as measurements are recorded more frequently, the covariance estimates become larger. This behavior is clearly incorrect, and arises due to the fact that the dependency between consecutive displacement estimates is ignored.

The preceding analysis substantiates, at least in the simple case of a robot moving in 1-D, that the use of expression (10) for covariance propagation results in considerably more accurate covariance estimates. Unfortunately, for robots moving in two dimensions [4] and three dimensions [2], the covariance propagation equations are time varying (the Jacobians appearing in (10) depend on the robot state and the positions of the features relative to the robot). As a result, an analogous closed-form analysis for general trajectories and arbitrary feature placement appears to be intractable. However, simulation experiments indicate that the conclusions drawn from the analytical expressions for the 1-D case also apply to the more practical scenarios of robots moving in two and three dimensions. For example, Fig. 3 shows the position and attitude covariance at the end of a 100-m trajectory for a robot performing visual odometry with a stereo pair of cameras [2]. The plotted lines represent the traces of the submatrices of the covariance matrix corresponding, respectively, to position (top subplot) and attitude (bottom subplot). These plots once again show that the covariance is a monotonically increasing function of measurement spacing when the exact expression of (10) is employed, while an artificial "valley" appears when the correlation terms in (10) are ignored.

\section{Filtering With Correlated Relative-State MEASUREMENTS}

We now describe the formulation of an EKF estimator that can fuse proprioceptive and relative-pose measurements, while properly accounting for the correlations in the latter. 

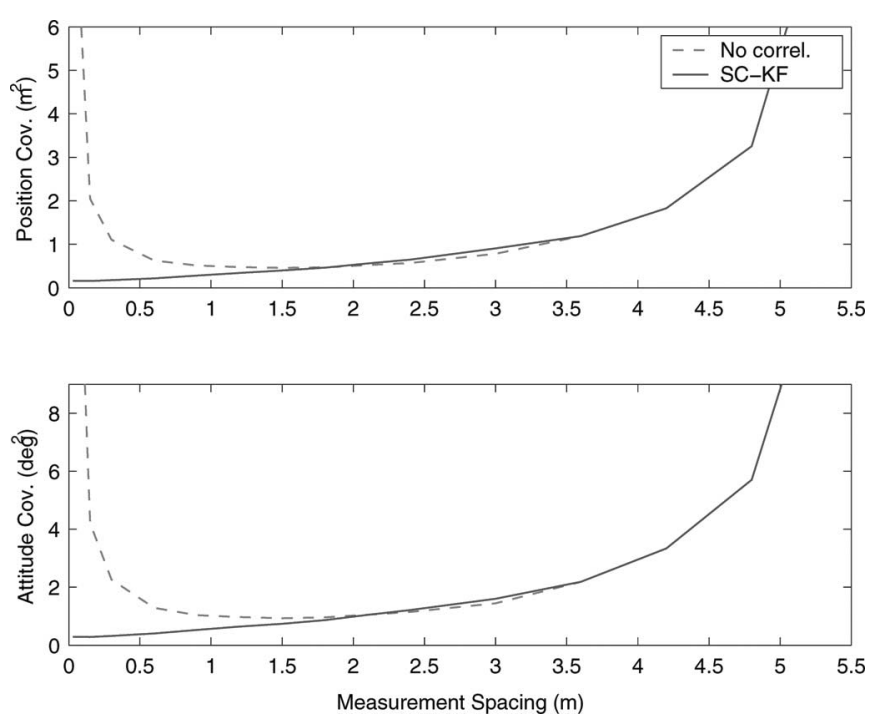

Fig. 3. Covariance estimates at the end of a 100-m trajectory for a robot performing visual odometry with a stereo pair. The top plot shows the position uncertainty, while the bottom one the attitude uncertainty. When correlations are properly treated (solid lines), the covariance is a monotonic function of the measurement spacing. This is not the case when correlations are ignored (dashed lines).

To reiterate the challenge posed in Section I, displacement measurements relate two robot states, and therefore, the joint probability density function ( $p d f$ ) of these states must be available in the filter. For this reason, we augment the EKF (error) state vector ${ }^{3}$ to include two copies of the robot's error state (cloning) [9]. The first copy of the error vector $\widetilde{X}_{k}$ represents the pose error at the instant when the latest exteroceptive measurement was recorded, while the second copy $\widetilde{X}_{k+i}$ represents the error in the robot's current state. In the propagation phase of the filter, only the current (evolving) state is propagated, while the previous state remains unchanged. Consequently, the robot states related by each displacement estimate are both represented explicitly in the filter state.

To correctly account for the correlations between consecutive relative-state measurements, the state vector is additionally augmented to include the errors of the latest exteroceptive measurement [11]. Thus, if the most recent exteroceptive measurement was recorded at $t_{k}$, the filter's error-state vector at $t_{k+i}$ is

$$
\breve{X}_{k+i \mid k}=\left[\begin{array}{lll}
\widetilde{X}_{k \mid k}^{T} & \widetilde{X}_{k+i \mid k}^{T} & \tilde{z}_{k+i \mid k}^{k T}
\end{array}\right]^{T}
$$

where the subscript $\ell \mid j$ denotes the value of a quantity at time $t_{\ell}$, after exteroceptive measurements up to time $t_{j}$, and proprioceptive measurements up to time $t_{\ell-1}$, have been processed. It is important to note that when odometry and displacement measurements are combined for pose estimation, it is possible to apply corrections to the exteroceptive measurements (cf. Section IV-C). Therefore, in the SC-KF, we also maintain an estimate $\widehat{z}_{k+i \mid k}^{k}$ of the most recent measurement. ${ }^{4}$ In this notation,

\footnotetext{
${ }^{3}$ Since the EKF is employed for estimation, the state vector comprises the errors in the estimated quantities, rather than the estimates. Therefore, cloning has to be applied to both the error states and the state estimates.

${ }^{4}$ To be more precise, this is an estimate of the physical quantities measured by the sensor, such as the distance and bearing to a set of features.
}

the superscript denotes the time instant at which the measurement was received, while the double subscript has the meaning explained before. The errors $\tilde{z}_{k+i \mid k}^{k}$ are defined accordingly.

By including the measurement error in the system's state vector, the dependency of the relative-state measurement $z_{k, k+i}$ on the exteroceptive measurement $z_{k}$ is transformed into a dependency on the current state of the filter, and the problem can now be treated in the standard EKF framework. It should be noted that since the measurement error is the source of the correlation between the current and previous displacement estimates, this is the "minimum-length" vector that must be appended to the state vector in order to incorporate the existing dependencies. Thus, this approach yields the minimal computational overhead needed to account for these correlations.

\section{A. Filter Initialization}

Consider the case where the first exteroceptive measurement $z_{0}$ is taken at time $t_{0}=0$, and let the robot's state estimate and covariance be denoted by $\widehat{X}_{0 \mid 0}$ and $P_{0 \mid 0}$, respectively. The initial error-state vector for the SC-KF contains the robot state and its clone, as well as the errors of the exteroceptive measurements at time $t_{0}$ [cf. (20)]:

$$
\breve{X}_{0 \mid 0}=\left[\begin{array}{lll}
\widetilde{X}_{0 \mid 0}^{s T} & \widetilde{X}_{0 \mid 0}^{T} & \widetilde{z}_{0 \mid 0}^{0 T}
\end{array}\right]^{T} .
$$

The superscript $s$ in (21) refers to the static copy of the state, which will remain unchanged during propagation.

Cloning of the robot state creates two identical random variables that convey the same information, and are thus, fully correlated. Moreover, since $z_{0}$ is not used to estimate the initial robot state, the latter is independent of the measurement errors at time $t_{0}$. Thus, the initial covariance matrix of the SC-KF state vector has the form

$$
\breve{P}_{0 \mid 0}=\left[\begin{array}{ccc}
P_{0 \mid 0} & P_{0 \mid 0} & 0 \\
P_{0 \mid 0} & P_{0 \mid 0} & 0 \\
0 & 0 & R_{0}
\end{array}\right]
$$

where 0 denotes a zero matrix of appropriate dimensions.

\section{B. State Propagation}

During regular operation, the filter's state covariance matrix, immediately after the relative-state measurement $z_{k-\ell, k}=$ $\xi\left(z_{k-\ell}, z_{k}\right)$ has been processed, takes the form

$$
\breve{P}_{k \mid k}=\left[\begin{array}{ccc}
P_{k \mid k} & P_{k \mid k} & P_{X_{k} z_{k}} \\
P_{k \mid k} & P_{k \mid k} & P_{X_{k} z_{k}} \\
P_{X_{k} z_{k}}^{T} & P_{X_{k} z_{k}}^{T} & P_{z_{k} z_{k}}
\end{array}\right]
$$

where $P_{k \mid k}$ is the covariance of the robot state at $t_{k}, P_{z_{k} z_{k}}$ is the covariance matrix of the error $\widetilde{z}_{k \mid k}^{k}$, and $P_{x_{k} z_{k}}=E\left\{\widetilde{X}_{k} \widetilde{z}_{k \mid k}^{k T}\right\}$ is the cross correlation between the robot's state and the measurement error at $t_{k}$ (closed-form expressions for $P_{z_{k} z_{k}}$ and $P_{X_{k} z_{k}}$ are derived in Section IV-C). Between two consecutive updates, proprioceptive measurements are employed to propagate the filter's state and its covariance. The robot's state estimate is 
propagated in time by the, generally nonlinear, equation

$$
\widehat{X}_{k+1 \mid k}=f\left(\widehat{X}_{k \mid k}, v_{k}\right)
$$

where $v_{k}$ denotes the proprioceptive (e.g., linear and rotational velocity) measurement at $t_{k}$. Linearization of (24) yields the error-propagation equation for the (evolving) second copy of the robot state

$$
\widetilde{X}_{k+1 \mid k} \simeq F_{k} \widetilde{X}_{k \mid k}+G_{k} \widetilde{v}_{k}
$$

where $F_{k}$ and $G_{k}$ are the Jacobians of $f\left(\widehat{X}_{k \mid k}, v_{k}\right)$ with respect to $\widehat{X}_{k \mid k}$ and $v_{k}$, respectively. Since the cloned state $X_{k \mid k}^{s}$ as well as the estimate for the measurement $z_{k}$ do not change with the integration of a new proprioceptive measurement, the error propagation equation for the augmented state vector is

$$
\breve{X}_{k+1 \mid k}=\breve{F}_{k} \breve{X}_{k \mid k}+\breve{G}_{k} \widetilde{v}_{k}
$$

with

$$
\breve{F}_{k}=\left[\begin{array}{ccc}
I & 0 & 0 \\
0 & F_{k} & 0 \\
0 & 0 & I
\end{array}\right] \quad \text { and } \quad \breve{G}_{k}=\left[\begin{array}{c}
0 \\
G_{k} \\
0
\end{array}\right]
$$

where $I$ denotes an identity matrix of appropriate dimensions. Thus, the covariance matrix of the propagated filter state is

$$
\begin{aligned}
\breve{P}_{k+1 \mid k} & =\breve{F}_{k} \breve{P}_{k \mid k} \breve{F}_{k}^{T}+\breve{G}_{k} Q_{k} \breve{G}_{k}^{T} \\
& =\left[\begin{array}{ccc}
P_{k \mid k} & P_{k \mid k} F_{k}^{T} & P_{X_{k} z_{k}} \\
F_{k} P_{k \mid k} & F_{k} P_{k \mid k} F_{k}^{T}+G_{k} Q_{k} G_{k}^{T} & F_{k} P_{X_{k} z_{k}} \\
P_{X_{k} z_{k}}^{T} & P_{X_{k} z_{k}}^{T} F_{k}^{T} & P_{z_{k} z_{k}}
\end{array}\right]
\end{aligned}
$$

where $Q_{k}=E\left\{\widetilde{v}_{k} \widetilde{v}_{k}^{T}\right\}$ is the covariance of the proprioceptive measurement $v_{k}$.

By straightforward calculation, if $m$ propagation steps occur between two consecutive relative-state updates, the covariance matrix $\breve{P}_{k+m \mid k}$ is determined as

$$
\breve{P}_{k+m \mid k}=\left[\begin{array}{ccc}
P_{k \mid k} & P_{k \mid k} \mathcal{F}_{k+m, k}^{T} & P_{X_{k} z_{k}} \\
\mathcal{F}_{k+m, k} P_{k \mid k} & P_{k+m \mid k} & \mathcal{F}_{k+m, k} P_{X_{k} z_{k}} \\
P_{X_{k} z_{k}}^{T} & P_{X_{k} z_{k}}^{T} \mathcal{F}_{k+m, k}^{T} & P_{z_{k} z_{k}}
\end{array}\right]
$$

where $\mathcal{F}_{k+m, k}=\prod_{i=0}^{m-1} F_{k+i}$, and $P_{k+m \mid k}$ is the propagated covariance of the robot state at $t_{k+m}$. The form of (29) shows that the covariance matrix of the filter can be propagated with minimal computation. In an implementation where efficiency is of utmost importance, the product $\mathcal{F}_{k+m, k}$ can be accumulated, and the matrix multiplications necessary to compute $\breve{P}_{k+m \mid k}$ can be delayed and carried out only when a new exteroceptive measurement is processed.

\section{State Update}

We next consider the state-update step of the SC-KF. Assume that a new exteroceptive measurement $z_{k+m}$ is recorded at $t_{k+m}$, and along with $\widehat{z}_{k+m \mid k}^{k}$, it is used to produce a relative-state measurement $z_{k, k+m}=\xi\left(\widehat{z}_{k+m \mid k}^{k}, z_{k+m}\right)$, relating robot poses $X_{k}$ and $X_{k+m}$. Note that $z_{k, k+m}$ may not fully determine all the degrees of freedom of the pose change between $t_{k}$ and $t_{k+m}$. For example, the scale is unobservable when using a single camera to estimate displacement via point-feature correspondences [8]. Thus, the relative-state measurement is equal to a nonlinear function of the robot poses at $t_{k}$ and $t_{k+m}$, with the addition of error

$$
z_{k, k+m}=h\left(X_{k}, X_{k+m}\right)+\widetilde{z}_{k, k+m} .
$$

The expected value of $z_{k, k+m}$ is computed from the state estimates at $t_{k}$ and $t_{k+m}$ as

$$
\widehat{z}_{k, k+m}=h\left(\widehat{X}_{k \mid k}, \widehat{X}_{k+m \mid k}\right)
$$

and therefore, based on (2), the innovation is given by

$$
\begin{aligned}
r_{k+m}= & z_{k, k+m}-\widehat{z}_{k, k+m} \simeq H_{k} \widetilde{X}_{k \mid k}+H_{k+m} \widetilde{X}_{k+m \mid k} \\
& +J_{k, k+m}^{k} \widetilde{z}_{k+m \mid k}^{k}+J_{k, k+m}^{k+m} \widetilde{z}_{k+m \mid k}^{k+m}+n_{k, k+m}
\end{aligned}
$$

where $H_{k}$ and $H_{k+m}$ are the Jacobians of $h\left(X_{k}, X_{k+m}\right)$ with respect to $X_{k}$ and $X_{k+m}$, respectively. We note that the quantity $\widetilde{z}_{k+m \mid k}^{k+m}$ appearing in the last equation is equal to the sensor noise in the measurement $z_{k+m}$, i.e., $\widetilde{z}_{k+m \mid k}^{k+m}=\widetilde{z}_{k+m}$.

In order to simplify the presentation of the state update equations, it is helpful to think of the displacement measurement $z_{k, k+m}$ as a constraint relating the robot poses $X_{k}$ and $X_{k+m}$ and the measurements $z_{k}$ and $z_{k+m}$. If we consider the "temporary" variable

then we can write (32) as

$$
X^{*}=\left[\begin{array}{ll}
\breve{X}_{k+m \mid k}^{T} & \widetilde{z}_{k+m \mid k}^{k+m T}
\end{array}\right]^{T}
$$

$$
\begin{aligned}
r_{k+m} & \simeq\left[\begin{array}{llll}
H_{k} & H_{k+m} & J_{k, k+m}^{k} & J_{k, k+m}^{k+m}
\end{array}\right] X^{*}+n_{k, k+m} \\
& =H X^{*}+n_{k, k+m} .
\end{aligned}
$$

This linearized residual expression can be used for carrying out an update on $X^{*}$ (and thus, on its constituent variables), using the standard EKF methodology. The covariance of the residual is

$$
\breve{S}_{k+m}=H P H^{T}+R_{n_{k, k+m}}
$$

where $R_{n_{k, k+m}}$ is the covariance of the noise term $n_{k, k+m}$ and

$$
P=\left[\begin{array}{cc}
\breve{P}_{k+m \mid k} & 0 \\
0 & R_{k+m}
\end{array}\right] .
$$

The Kalman gain for updating $X^{*}$ is given by

$$
K=P H^{T} \breve{S}_{k+m}^{-1}=\left[\begin{array}{llll}
K_{k}^{T} & K_{k+m}^{T} & K_{z_{k}}^{T} & K_{z_{k+m}}^{T}
\end{array}\right]^{T}
$$

where $K_{k}, K_{k+m}, K_{z_{k}}$, and $K_{z_{k+m}}$ are the block elements of $K$ corresponding to $X_{k}, X_{k+m}, z_{k}$, and $z_{k+m}$, respectively. We note that although the measurement $z_{k+m}$ can be used to update the robot's pose at $t_{k}$ and the previous measurement $z_{k}$, these variables will no longer be needed, so we can omit computation of $K_{k}$ and $K_{z_{k}}$. Only the block elements $K_{k+m}$ and $K_{z_{k+m}}$ need to be evaluated. Taking into consideration the special structure of $H$ and $P$, we obtain

$$
\begin{aligned}
K_{k+m}= & \left(\mathcal{F}_{k+m, k} P_{k \mid k} H_{k}^{T}+P_{k+m \mid k} H_{k+m}^{T}\right. \\
& \left.+\mathcal{F}_{k+m, k} P_{X_{k} z_{k}} J_{k, k+m}^{k T}\right) \breve{S}_{k+m}^{-1} \\
K_{z_{k+m}}= & R_{k+m} J_{k, k+m}^{k+m T} \breve{S}_{k+m}^{-1} .
\end{aligned}
$$


Using these results, the equations for updating the current robot state and the measurements $z_{k+m}$ are

$$
\begin{aligned}
\widehat{X}_{k+m \mid k+m} & =\widehat{X}_{k+m \mid k}+K_{k+m} r_{k+m} \\
\widehat{z}_{k+m \mid k+m}^{k+m} & =z_{k+m}+K_{z_{k+m}} r_{k+m} .
\end{aligned}
$$

After $z_{k, k+m}$ is processed, the clone of the previous state error $\widetilde{X}_{k \mid k}$ and the previous measurement error $\widetilde{z}_{k+m \mid k}^{k}$ are discarded. The robot's current state $X_{k+m \mid k+m}$ is cloned, and the updated exteroceptive measurement errors $\widetilde{z}_{k+m \mid k+m}^{k+m}$ are appended to the new filter state.

Thus, the filter error-state vector becomes

$$
\breve{X}_{k+m \mid k+m}=\left[\begin{array}{lll}
\widetilde{X}_{k+m \mid k+m}^{T} & X_{k+m \mid k+m}^{T} & \widetilde{z}_{k+m \mid k+m}^{k+m T}
\end{array}\right]^{T} .
$$

The state update process is completed by computing the covariance matrix of $\breve{X}_{k+m \mid k+m}$. To this end, we note that the covariance matrix of $X^{*}$ is updated as $P \leftarrow P-K \breve{S}_{k+m} K^{T}$. Using the structure of the matrices involved in this equation, we obtain

$$
\breve{P}_{k+m \mid k+m}=\left[\begin{array}{lll}
P_{k+m \mid k+m} & P_{k+m \mid k+m} & P_{X_{k+m} z_{k+m}} \\
P_{k+m \mid k+m} & P_{k+m \mid k+m} & P_{X_{k+m}} z_{k+m} \\
P_{X_{k+m} z_{k+m}}^{T} & P_{X_{k+m} z_{k+m}}^{T} & P_{z_{k+m}} z_{k+m}
\end{array}\right]
$$

where

$$
\begin{gathered}
P_{k+m \mid k+m}=P_{k+m \mid k}-K_{k+m} \breve{S}_{k+m} K_{k+m}^{T} \\
P_{z_{k+m}} z_{k+m}=R_{k+m}-R_{k+m} J_{k, k+m}^{k+m T} \breve{S}_{k+m}^{-1} J_{k, k+m}^{k+m} R_{k+m} \\
P_{X_{k+m} z_{k+m}}=-K_{k+m} J_{k, k+m}^{k+m} R_{k+m} .
\end{gathered}
$$

For clarity, the steps of the SC-KF algorithm are outlined in Algorithm 2.

\section{Algorithm 2: Stochastic Cloning Kalman Filter}

Initialization: When the first exteroceptive measurement is received:

- clone the state estimate $\widehat{X}_{0 \mid 0}$

- initialize the filter state covariance matrix using (22)

Propagation: For each proprioceptive measurement:

- propagate the evolving copy of the robot state via (24)

- propagate the filter covariance using (28), or equivalently (29)

Update: For each exteroceptive measurement:

- compute the relative-state measurement using (1), and its Jacobians with respect to the current and previous exteroceptive measurement, using (3).

- update the current robot state using equations (31), (32), (34), (36), and (38)

- update the current measurement using (37) and (39)

- remove the previous robot state and exteroceptive measurement

- create a cloned copy of the current robot state

- compute the covariance of the new augmented state vector [cf. (40)] using (41)-(44)

\section{Computational Complexity}

While our proposed state-augmentation approach does account for the correlations that have been neglected in previous work, its use imposes a small additional cost in terms of computation and memory requirements. We now show that these algorithmic requirements are linear in the number of features observed at a single time step.

If $N$ and $M_{k}$, respectively, denote the dimensions of the robot's state and the size of the measurement vector at $t_{k}$, then the covariance matrix $\breve{P}_{k+m \mid k}$ has size $\left(2 N+M_{k}\right) \times(2 N+$ $M_{k}$ ). If $M_{k} \gg N$, the overhead of state augmentation is mostly due to the inclusion of the measurements in the filter state vector, which leads to the correct treatment of the temporal correlations in the relative-pose measurements. If these correlations are ignored, the size of the filter state vector is twice the size of the robot's state vector. In this case, the computational complexity and memory requirements are $O\left(N^{2}\right)$. In the algorithm proposed in this paper, the most computationally expensive operation for $M_{k} \gg N$ is the evaluation of the covariance of the residual $\breve{S}_{k+m}$ [cf. (34)]. The covariance matrix $\breve{P}_{k+m \mid k}$ is of dimensions $\left(2 N+M_{k}\right) \times\left(2 N+M_{k}\right)$, and thus, the computational complexity of obtaining $\breve{S}_{k+m}$ is, generally, $O((2 N+$ $\left.\left.M_{k}\right)^{2}\right) \approx O\left(M_{k}^{2}\right)$. However, from (43), we see that the submatrix $P_{z_{k} z_{k}}$ of $\breve{P}_{k+m \mid k}$, which corresponds to the updated measurement covariance matrix, has the following structure:

$$
P_{z_{k} z_{k}}=\underbrace{R_{k}}_{M_{k} \times M_{k}}-\underbrace{R_{k} J_{k-m, k}^{k T}}_{M_{k} \times N} \underbrace{\breve{S}_{k}^{-1}}_{N \times N} \underbrace{J_{k-m, k}^{k} R_{k}}_{N \times M_{k}} .
$$

As explained in Section III, the measurement noise covariance matrix $R_{k}$ is commonly block diagonal. Therefore, $P_{z_{k} z_{k}}$ has the special structure of a block-diagonal matrix minus a rank- $N$ update. By exploiting this structure when evaluating (34), the operations needed reduce to $O\left(N^{2} M_{k}\right)$. Moreover, the submatrix $P_{z_{k} z_{k}}$ does not need to be explicitly formed, which decreases the storage requirements of the algorithm to $O\left(N^{2}+N M_{k}\right) \approx O\left(N M_{k}\right)$. For more details on this point, the interested reader is referred to [18].

Furthermore, for a number of applications, it is not necessary to maintain a clone of the entire robot state and its covariance. Close inspection of the filter update equations reveals that only the states that directly affect the relative-state measurement (i.e., those that are needed to compute the expected relative-state measurement and its Jacobians) are required for the update step. The remaining states and their covariance need not be cloned, thus further reducing the memory and computational requirements. For example, when measurements from an inertial measurement unit (IMU) are employed for localization, estimates for the bias of the IMU measurements are often included in the state vector [19]. These bias estimates clearly do not appear in (31), and therefore, it is not necessary to maintain their clones in the filter.

\section{EXTENSIONS}

\section{A. Treatment of Additional Measurements}

To simplify the presentation, in the previous section, it was assumed that only proprioceptive and relative-pose measurements are available. However, this assumption is not necessary, 
as additional measurements can be processed in the standard EKF methodology [20]. For example, let

$$
z_{k+\ell}=\zeta\left(X_{k+\ell}\right)+n_{k+\ell}
$$

be an exteroceptive measurement received at $t_{k+\ell}$. By linearizing, we obtain the measurement error equation as follows:

$$
\begin{aligned}
\widetilde{z}_{k+\ell} & =H_{k+\ell}^{\prime} \widetilde{X}_{k+\ell \mid k}+n_{k+\ell} \\
& =\left[\begin{array}{lll}
0 & H_{k+\ell}^{\prime} & 0
\end{array}\right]\left[\begin{array}{c}
\widetilde{X}_{k \mid k} \\
\widetilde{X}_{k+\ell \mid k} \\
\widetilde{z}_{k+\ell \mid k}^{k}
\end{array}\right]+n_{k+\ell} .
\end{aligned}
$$

Since this expression adheres to the standard EKF model, the augmented filter state can be updated without any modifications to the algorithm. However, if additional measurements are processed, the compact special expressions of (29) and (36) are no longer valid, as update steps occur between consecutive displacement estimates. In this case, the general form of the SC-KF equations must be used.

Another practically important case occurs when more than one sensor provides relative-pose measurements, but at different rates. Such a situation would arise, for example, when a mobile robot is equipped with a camera and a laser range finder. In such a scenario, the state-augmentation approach of the SC-KF still applies. In particular, every time either of the sensors records a measurement, cloning is applied. Therefore, at any given time, the filter state vector comprises: 1) three instances of the robot state, corresponding to the current state, and the state at the last time instants where each sensor received a measurement, and 2) the errors in the latest exteroceptive measurement of each sensor. Although the propagation and update equations must be modified to account for the change in dimension of the state vector, the basic principles of the approach still apply.

\section{B. Extension To Multiple States}

In the algorithm presented in Section IV, feature measurements are processed to construct displacement estimates, which subsequently define constraints between consecutive robot poses. By including two robot poses in the filter state vector, the SC-KF can optimally process successive exteroceptive measurements, while incurring a computational cost linear in the number of observed features. However, when a static feature is observed more than two times, the basic SC-KF must be modified. Intuitively, the observation of a static feature from multiple robot poses should impose a geometric constraint involving these measurements and all of the corresponding poses. We now briefly describe an extension to the SC-KF approach that correctly incorporates multiple observations of a single point feature while still maintaining computational complexity linear in the number of locally observed features [21].

Let $Y_{f_{j}}$ be the position of a static feature that is observed from $L \geq 2$ consecutive robot poses $X_{k}, X_{k+1}, \ldots, X_{k+L-1}$. The measurement function $h_{f_{j}}$ corresponding to these measurements is

$$
z_{k+i}^{f_{j}}=h_{f_{j}}\left(X_{k+i}, Y_{f_{i}}\right)+n_{k+i}^{f_{j}}, \quad \text { for } \quad i=0, \ldots, L-1
$$

where $n_{k+i}^{f_{j}}$ is the measurement noise. Stacking these $L$ equations results in a block measurement equation of the form

$$
\mathbf{z}_{f_{j}}=\mathbf{h}_{f_{j}}\left(X_{k}, X_{k+1}, \ldots, X_{k+L-1}, Y_{f_{j}}\right)+\mathbf{n}_{f_{j}} .
$$

Eliminating the feature position $Y_{f_{j}}$ from (47) yields a constraint vector that involves all of the robot poses:

$$
\mathbf{c}_{f_{j}}\left(X_{k}, X_{k+1}, \ldots, X_{k+L-1}, \mathbf{z}_{f_{j}}, \mathbf{n}_{f_{j}}\right)=\mathbf{0}_{q}
$$

where $q$ is the dimension of the constraint vector $\mathbf{c}_{f_{j}}$. If the EKF state vector has been augmented to include the $L$ copies of the robot pose, this equation can be used to perform an EKF update, thus utilizing all the geometric information provided by the observations of this feature. Furthermore, if $M_{k}$ features are observed from $L$ robot poses, then a constraint vector $\mathbf{c}_{f_{j}}, j=1, \ldots, M_{k}$, can be written for each of these features. Since the feature measurements are mutually uncorrelated, the resulting constraints will also be uncorrelated, and therefore, an EKF update that utilizes all $M_{k}$ constraints can be performed in $O\left(M_{k}\right)$ time.

\section{RELATION TO SLAM}

An alternative approach to processing the feature measurements obtained with an exteroceptive sensor is to jointly estimate the robot's pose and the feature positions. This is the well-known SLAM problem, which has been extensively studied (e.g., [22]-[25]). This section examines the relation of the SC-KF algorithm to SLAM. Enumerated as follows are some important points:

1) Computational Complexity: If an exact solution to SLAM was possible, the resulting pose estimates would be optimal, since all the positioning information would be used and all the interdependencies between the robot and the feature states would be accounted for. However, good localization performance comes at a considerable computational cost. It is well known that the computational complexity and memory requirements of the EKF solution to SLAM increase quadratically with the total number of features in the environment [22]. While several approximate solutions exist that possess lower computational complexity (e.g., [23], [25], and [26]), many of them cannot guarantee the consistency of the estimates, nor is there a concrete measure of suboptimality.

Since the high computational burden of SLAM is due to the need to maintain a map of the environment, the amount of computational resources allocated for localization constantly increases as the robot navigates in an unknown environment. For continual operation over an extended period, this overhead can become unacceptably large. Even in an approximate SLAM algorithm, the largest portion of the computational resources is devoted to maintaining the constantly expanding feature map. However, there exist a number of applications where building a map is not necessary, while real-time performance is of utmost importance (e.g., in autonomous aircraft landing [27] or emergency response [28]). Such applications require high localization accuracy, but with minimal computational overhead.

The SC-KF uses pairs of consecutive exteroceptive measurements to produce displacement estimates, which are then 
fused with proprioceptive sensing information. As shown in Section IV-D, our algorithm's complexity is linear in the number of features observed only at each time step. In most cases, this number is orders of magnitude smaller than the total number of features in the environment. A reduced-complexity SLAM approach that is similar in spirit to the SC-KF would consist of maintaining only the most recently acquired local features, i.e., those that are currently visible by the robot, in the state vector. However, the algorithmic complexity of such an EKF-SLAM would be quadratic in the number of local features. In contrast, the SC-KF is linear in the number of local features.

2) Feature Position Observability: SLAM algorithms require the states of the local features to be completely observable, in order to be included in the state vector. When a single measurement does not provide sufficient information to initialize a feature's position estimate with bounded uncertainty, feature initialization schemes must be implemented [29], [30]. In fact, state augmentation is an integral part of many methods for delayed feature initialization [31], [32]. In contrast, in the SC-KF framework, feature initialization is not required since the feature measurements are included in the augmented state vector, instead of the feature positions.

3) Data Association: Since only pairs of exteroceptive measurements are used by the SC-KF algorithm, the data association problem is simplified. In contrast, SLAM requires a correspondence search over all map features in the robot's vicinity, and its computational overhead is considerably higher [33]. To facilitate robust data association, it is a common practice to employ a feature-detection algorithm that extracts "high-level" features (e.g., landmarks such as corners, junctions, and straight-line segments) from raw sensor data. Then, only these features are employed for SLAM.

4) Information Loss: While the extraction of high-level features results in more robust and computationally tractable algorithms (e.g., laser scans consist of hundreds of range points, but may contain only a few corner features), this approach effectively discards information contained in the sensor data (cf. Fig. 1). Consequently, the resulting estimates of the robot's pose are suboptimal compared to those that use all the available information. Maintaining and processing the entire history of raw sensor input (e.g., [34]) can lead to excellent localization performance, but such an approach may be infeasible for real-time implementation on typical mobile robots. A benefit of the SC-KF approach is that it takes advantage of all the available information in two consecutive exteroceptive measurements (i.e., most laser points in two scans can be used to estimate displacement by scan matching).

5) SC-KF and SLAM: For longer robot traverses, the positioning accuracy obtained when only pairs of exteroceptive measurements are considered is inferior to that of SLAM, as no loop closing occurs. Essentially, the SC-KF approach offers an "enhanced" form of DR, in the sense that the uncertainty of the robot's state monotonically increases over time. The rate of uncertainty increase, though, is significantly lower than that attained when only proprioceptive measurements are used (cf. Section VII). However, as mentioned in Section IV-D, in the SC-KF approach, the state vector $X_{k}$ is not required to contain only the robot pose. If high-level, stable features (landmarks) are available, their positions can be included in the "robot" state vector $X_{k}$. Therefore, the SC-KF method for processing relative-state measurements can be expanded and integrated with the SLAM framework. This integration would further improve the attainable localization accuracy within areas with lengthy loops. Since this modification is beyond the scope of this paper, in the following section, we present experimental results of applying the SC-KF algorithm to the case where only relative-state and proprioceptive measurements are considered.

\section{EXPERIMENTAL RESULTS}

This section presents experimental results that demonstrate the performance of the algorithms described in Sections III-A and IV. The experiments use a Pioneer II mobile robot equipped with a SICK LMS-200 laser rangefinder. The robot's pose consists of its planar position and orientation in a global frame:

$$
X_{k}=\left[\begin{array}{lll}
{ }^{G} x_{k} & { }^{G} y_{k} & { }^{G} \phi_{k}
\end{array}\right]^{T}=\left[\begin{array}{ll}
{ }^{G} p_{k}^{T} & { }^{G} \phi_{k}
\end{array}\right]^{T} .
$$

We first present results from the application of the SC-KF, and then, study the case where the robot's state is propagated based on displacement estimates exclusively (i.e., no proprioceptive measurements are processed).

\section{A. Stochastic Cloning Kalman Filter}

In this experiment, odometry measurements are fused with displacement measurements that are obtained by laser scan matching with the method presented in [6]. The SC-KF equations for the particular odometry and measurement model are presented in [18].

1) Experiment Description: During the first experiment, the robot traversed a trajectory of approximately $165 \mathrm{~m}$, while recording 378 laser scans. The robot processed a new laser scan approximately every $1.5 \mathrm{~m}$, or every time its orientation changed by $10^{\circ}$. Here, we compare the performance of the SC$\mathrm{KF}$ algorithm to that obtained by Hoffman et al. [10]. In [10], the displacement estimates and the previous pose estimates are combined to yield pseudomeasurements of the robot's absolute position. In order to guarantee consistent estimates for the latter case, we have employed the covariance intersection (CI) method [35] for fusing the pseudomeasurements of absolute position with the most current pose estimates. From here on, we refer to this approach as "pseudoabsolute updates."

As discussed in Section IV-D, the SC-KF has computational complexity linear in the number of feature measurements taken at each pose. If even this computational complexity is deemed too high for a particular application, one can ignore the correlations between consecutive displacement measurements at the expense of optimality. In that case, the augmented state only contains two copies of the robot state [9]. Results for this approximate, though computationally simpler, variant of the SC$\mathrm{KF}$, referred to as SC-KF-NC (i.e., no correlations between the measurement errors are considered), are also presented, and are compared with the performance of the SC-KF.

The robot trajectories estimated by the different algorithms are shown in Fig. 4. Fig. 5 presents the covariance estimates 


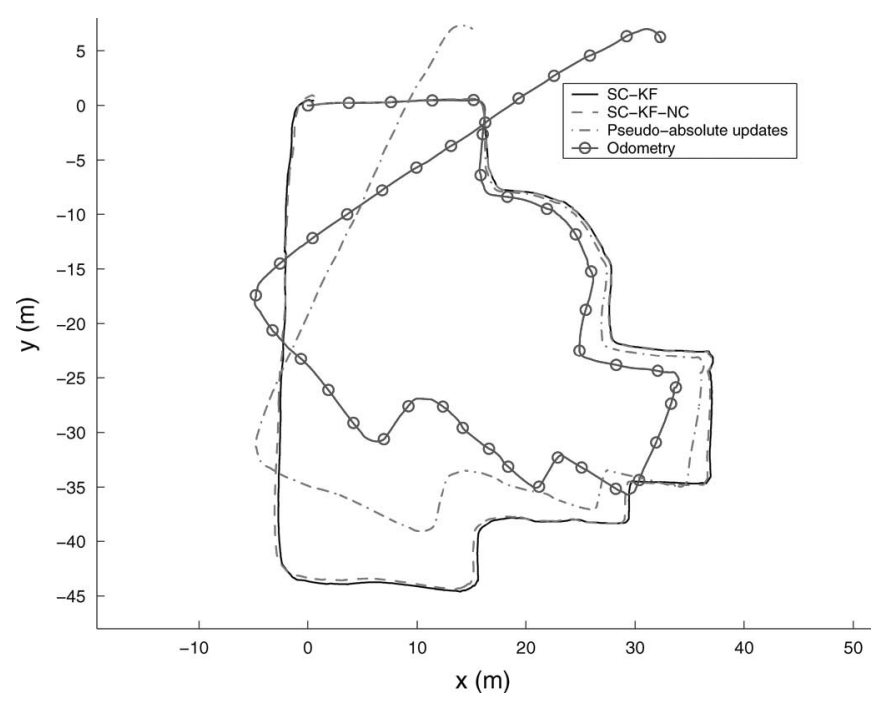

Fig. 4. Estimated trajectory of the robot using the SC-KF algorithm (solid line), the SC-KF-NC algorithm (dashed line), the method of [10] that uses absolute position pseudomeasurements (dash-dotted line), and odometry only (solid line with circles).

for the robot pose as a function of time. We observe that correctly accounting for the correlations between consecutive displacement estimates in the SC-KF results in smaller covariance values. Even though ground truth for the entire trajectory is not known, the final robot pose is known to coincide with the initial one. The errors in the final robot pose are equal to $\widetilde{X}=\left[\begin{array}{lll}0.5 \mathrm{~m} & 0.44 \mathrm{~m} & -0.11^{\circ}\end{array}\right]^{T}(0.4 \%$ of the trajectory length) for the SC-KF, $\widetilde{X}=\left[\begin{array}{lll}0.61 \mathrm{~m} & 0.65 \mathrm{~m} & -0.13^{\circ}\end{array}\right]^{T}$ $(0.54 \%$ of the trajectory length) for the SC-KF-NC, $\widetilde{X}=$ $\left[15.03 \mathrm{~m} \quad 7.07 \mathrm{~m}-32.3^{\circ}\right]^{T}$ (10.6\% of the trajectory length) for the approach in [10], and $\widetilde{X}=\left[\begin{array}{lll}32.4 \mathrm{~m} & 5.95 \mathrm{~m} & -69.9^{\circ}\end{array}\right]^{T}$ ( $19.9 \%$ of the trajectory length) for DR based on odometry. From these error values, as well as from visual inspection of the trajectory estimates in Fig. 4, we conclude that both the SC-KF and the SC-KF-NC yield very similar results. However, the approach based on creating pseudomeasurements of the absolute pose [10] performs significantly worse. It should be noted that the errors in the final robot pose are consistent with the estimated covariance in all cases considered.

2) Impact of Correlations: Clearly, the lack of ground truth data along the entire trajectory for the real-world experiment does not allow for a detailed comparison of the performance of the SC-KF and SC-KF-NC algorithms, as both appear to attain comparable estimation accuracy. Simulations are used to perform a more thorough assessment of the impact of the measurement correlations on the position accuracy and the uncertainty estimates. The primary objective of these simulations is to contrast the magnitude of the estimation errors with the computed covariance values in the cases when the correlations between consecutive measurements are accounted for (SC-KF) vs. when they are ignored (SC-KF-NC).

For the simulation results shown here, a robot moves in a circular trajectory of radius $4 \mathrm{~m}$, while observing a wall that lies $6 \mathrm{~m}$ from the center of its trajectory. The relative-pose measurements in this case are created by performing line matching, instead of point matching between consecutive scans [36]. Since only one line is available, the motion of the robot along the line direction is unobservable. As a result, the singular value decomposition of the covariance matrix of the robot's displacement estimate can be written as

$$
R_{k, k+m}=\left[V_{u} V_{o}\right]\left[\begin{array}{ccc}
s_{1} & 0 & 0 \\
0 & s_{2} & 0 \\
0 & 0 & s_{3}
\end{array}\right]\left[\begin{array}{c}
V_{u}^{T} \\
V_{o}^{T}
\end{array}\right], s_{1} \rightarrow \infty
$$

where $V_{u}$ is the basis vector of the unobservable direction (i.e., a unit vector along the direction of the wall, expressed with respect to the robot frame at time $t_{k}$ ) and $V_{o}$ is a $3 \times 2$ matrix, whose column vectors form the basis of the observable subspace. To avoid numerical instability in the filter, the displacement measurements $z_{k, k+m}$ computed by line matching are projected onto the observable subspace, thus creating a relative-state measurement of dimension 2, given by $z_{k, k+m}^{\prime}=V_{o}^{T} z_{k, k+m}$.

Fig. 6 shows the robot pose errors (solid lines), along with the corresponding 99.8th percentile of their distribution (dashed lines). The left column shows the results for the SC-KF algorithm presented in Section IV, while the right one for the SC-KF$\mathrm{NC}$ algorithm. As evident from Fig. 6, the covariance estimates of the SC-KF-NC are not commensurate with the corresponding errors. When the temporal correlations of the measurements are properly treated, as is the case for the SC-KF, substantially more accurate covariance estimates, which reflect the true uncertainty of the robot's state, are computed. Moreover, the evaluation of the rms value of the pose errors shows that the errors associated with the SC-KF algorithm (which accounts for correlations) are $25 \%$ smaller than those of the SC-KF-NC.

\section{B. State Propagation Based on Displacement Estimates}

We now present results for the case in which the robot's pose is estimated by using only displacement estimates computed from laser scan matching. Given a displacement estimate $z_{k, k+m}=$ $\left[{ }^{k} \widehat{p}_{k+m}^{T}{ }^{k} \widehat{\phi}_{k+m}\right]^{T}$, the global robot pose is propagated using the equations

$$
\begin{aligned}
& \widehat{X}_{k+m}=g\left(\widehat{X}_{k}, \widehat{z}_{k, k+m}\right) \\
& \Rightarrow\left[\begin{array}{l}
G \widehat{p}_{k+m} \\
{ }^{G} \widehat{\phi}_{k+m}
\end{array}\right]=\left[\begin{array}{l}
G \widehat{p}_{k} \\
{ }^{G} \widehat{\phi}_{k}
\end{array}\right]+\left[\begin{array}{c}
C\left({ }^{G} \widehat{\phi}_{k}\right)^{k} \widehat{p}_{k+m} \\
k \widehat{\phi}_{k+m}
\end{array}\right]
\end{aligned}
$$

where $C(\cdot)$ denotes the $2 \times 2$ rotation matrix. In this case, the Jacobian matrices $\Phi_{k}$ and $\Gamma_{k}$ are given by

$$
\begin{aligned}
\Phi_{k} & =\left[\begin{array}{cc}
I & -\Psi C\left({ }^{G} \widehat{\phi}_{k}\right)^{k} \widehat{p}_{k+m} \\
0 & 1
\end{array}\right], \quad \Psi=\left[\begin{array}{cc}
0 & -1 \\
1 & 0
\end{array}\right] \\
\Gamma_{k} & =\left[\begin{array}{cc}
C\left(G \widehat{\phi}_{k}\right) & 0 \\
0 & 1
\end{array}\right] .
\end{aligned}
$$

Fig. 7 presents the estimated robot trajectory, along with the map of the area that has been constructed by overlaying all the scan points, transformed using the estimates of the robot pose (we stress that the map is only plotted for visualization purposes, and is not estimated by the algorithm). This experiment used the same dataset from Section VII-A. Fig. 8 presents the 

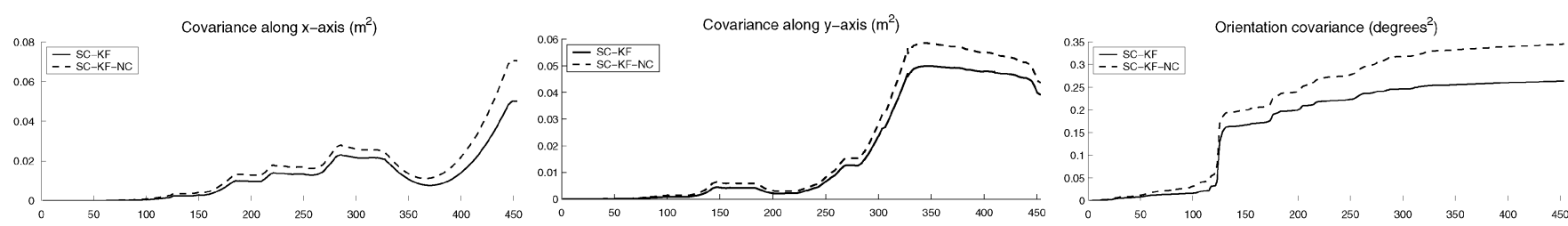

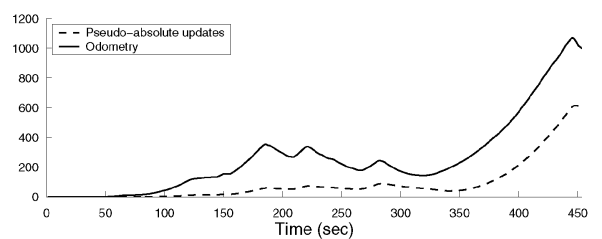

(a)

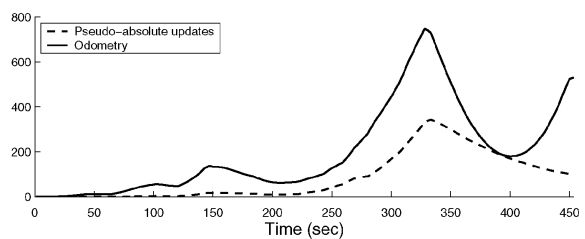

(b)

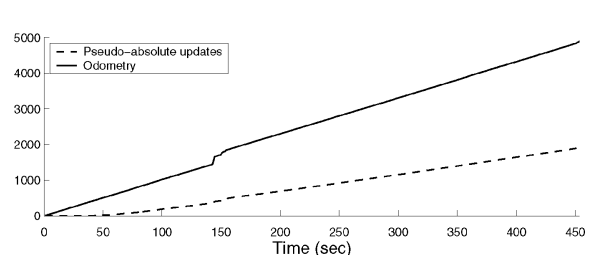

(c)

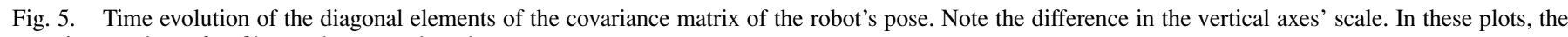
covariance values after filter updates are plotted.

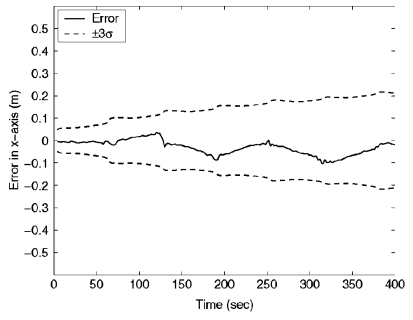

(a)

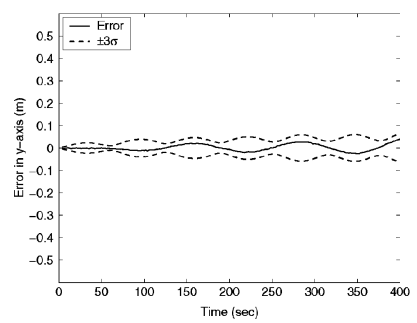

(c)

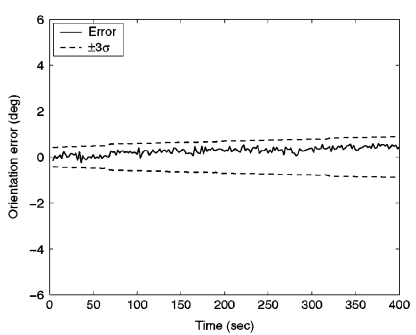

(e)

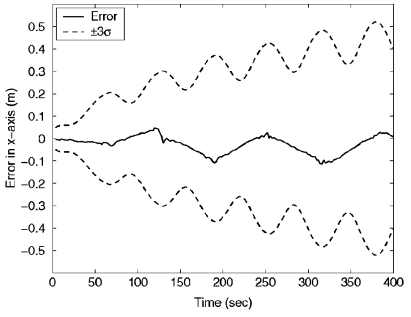

(b)

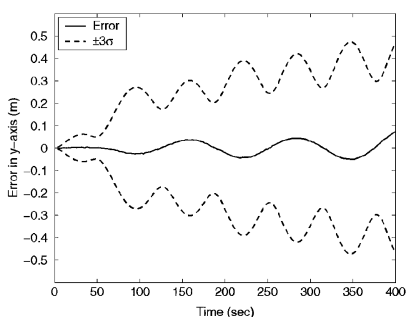

(d)

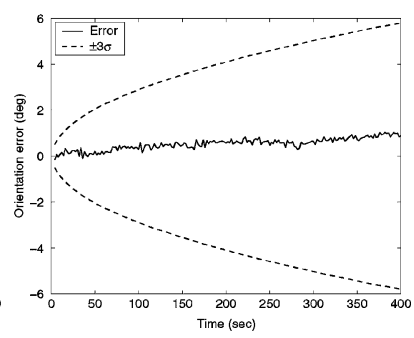

(f)

Fig. 6. Robot pose errors (solid lines) vs. the corresponding 99.8th percentile of their distribution (dashed lines). The left column shows the results for the $\mathrm{SC}-\mathrm{KF}$ algorithm proposed in this paper, while the right one demonstrates the results for the SC-KF-NC algorithm. In these plots, the covariance values after filter updates are plotted. (a) and (b) Errors and $\pm 3 \sigma$ bounds along the $x$-axis. (c) and (d) Errors and $\pm 3 \sigma$ bounds along the $y$-axis. (e) and (f) Orientation errors and $\pm 3 \sigma$ bounds.

covariance estimates for the robot's pose, computed by using (10) (SC-KF, solid lines) in contrast with those computed when the correlations between the consecutive displacement estimates are ignored (SC-KF-NC, dashed lines). As expected, the pose covariance is larger when only displacement measurements are used, compared to the case where odometry measurements are

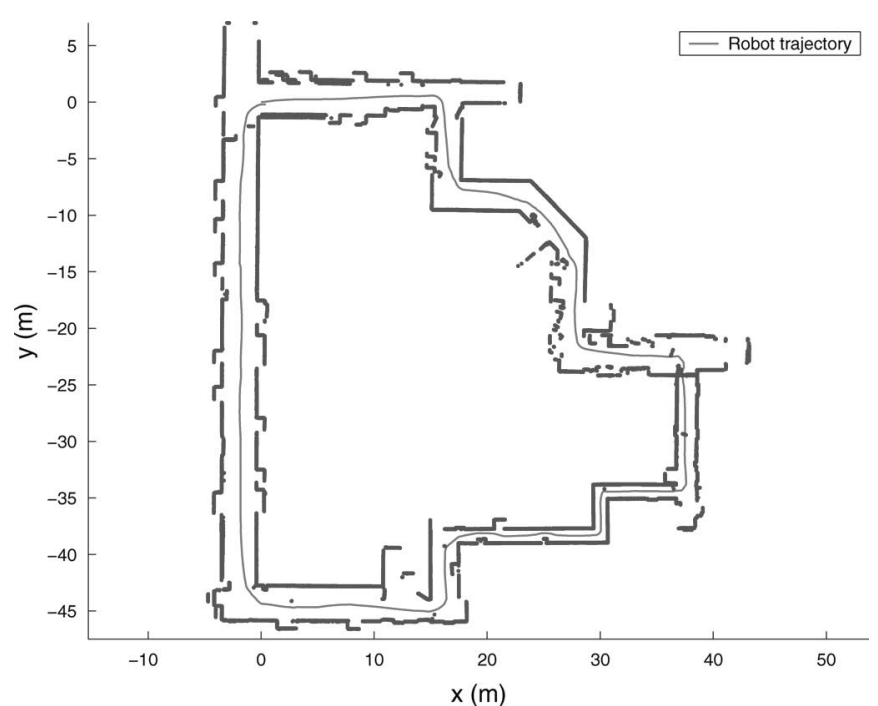

Fig. 7. Estimated trajectory of the robot based only on laser scan matching. The map is presented for visualization purposes only, by transforming all the laser points using the estimated robot pose. Some "spurious" points in the map are due to the presence of people.

fused with displacement measurements (cf. Fig. 5). From Fig. 8, we also observe that accounting for the correlations results in significantly smaller values for the estimated covariance of the robot pose, thus corroborating the discussion of Section III-B.

\section{CONCLUSION}

In this paper, we have proposed an efficient EKF-based estimation algorithm, termed SC-KF, for the problem of fusing proprioceptive measurements with relative-state measurements that are inferred from exteroceptive sensory input. An analysis of the structure of the measurement equations demonstrated that when the same exteroceptive measurements are processed to estimate displacement in consecutive time intervals, the displacement errors are temporally correlated. The main contribution of this paper is the introduction of a novel feature-marginalization process that allows for the processing of relative-pose measurements while also considering the correlations between these. This method is based on augmenting the state vector of the 


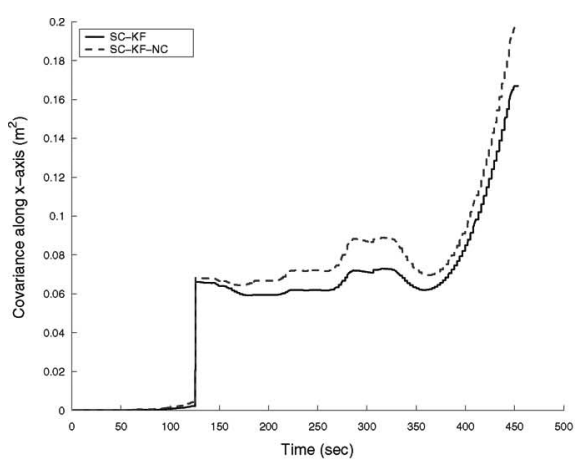

(a)

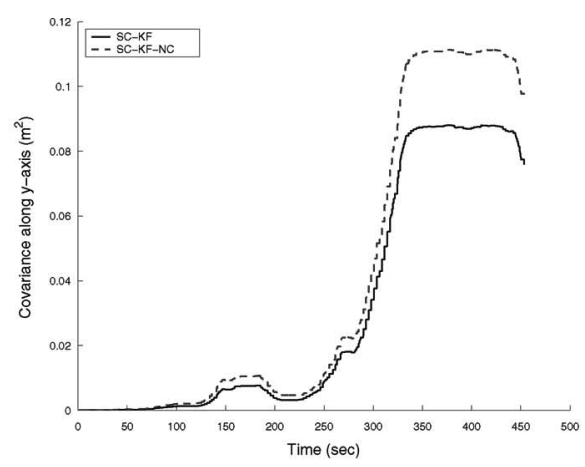

(b)

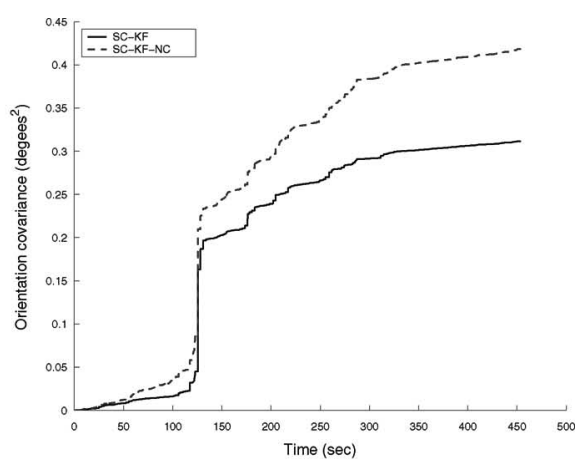

(c)

Fig. 8 Estimated covariance of the robot's pose when the correlation between consecutive measurements is properly accounted for (solid lines) versus the covariance estimated when the correlations are ignored (dashed lines). (a) Covariance along the $x$-axis. (b) Covariance along the $y$-axis. (c) Orientation covariance. At approximately $130 \mathrm{~s}$, a displacement estimate based on very few laser points was computed, resulting in a sudden increase in the covariance.

EKF to temporarily include the robot poses and the feature observations related through a local geometric constraint (i.e., a relative-state measurement). By employing state augmentation, the dependence of the relative-state measurement on previous states and measurements is transformed to a dependence on the current state of the filter, and this enables application of the standard EKF framework.

The experimental and simulation results demonstrate that the SC-KF method attains better localization performance compared to previous approaches [10], while the overhead imposed by the additional complexity is minimal. The method yields more accurate estimates, and most significantly, it provides a more precise description of the uncertainty in the robot's state estimates. Additionally, the method is versatile, since it is independent of the actual sensing modalities used to obtain the proprioceptive and exteroceptive measurements.

\section{REFERENCES}

[1] A. Kelly, "General solution for linearized systematic error propagation in vehicle odometry," in Proc. IEEE/RSJ Int. Conf. Intell. Robots Syst., Maui, HI, Oct. 29-Nov. 3, 2001, pp. 1938-1945.

[2] L. Matthies, "Dynamic stereo vision, " Ph.D. dissertation, Dept. Comput. Sci., Carnegie Mellon Univ., Pittsburgh, PA, 1989.

[3] C. Olson, L. Matthies, H. Schoppers, and M. Maimone, "Robust stereo ego-motion for long distance navigation," in Proc. CVPR, 2000, pp. $453-$ 458.

[4] F. Lu and E. Milios, "Robot pose estimation in unknown environments by matching 2D range scans," J. Intell. Robot. Syst.: Theory Appl., vol. 18, no. 3, pp. 249-275, Mar. 1997.

[5] D. Silver, D. M. Bradley, and S. Thayer, "Scan matching for flooded subterranean voids," in Proc. IEEE Conf. Robot., Autom. Mechatron. (RAM), Dec. 2004, pp. 422-427.

[6] S. T. Pfister, K. L. Kriechbaum, S. I. Roumeliotis, and J. W. Burdick, "Weighted range sensor matching algorithms for mobile robot displacement estimation," in Proc. IEEE Int. Conf. Robot. Autom., Washington, DC, May 11-15, 2002, pp. 1667-1674.

[7] K. Konolige, "Large-scale map-making," in Proc. AAAI Nat. Conf. Artif. Intell., San Jose, CA, Jul. 2004, pp. 457-463.

[8] P. Torr and D. Murray, "The development and comparison of robust methods for estimating the fundamental matrix," Int. J. Comput. Vis., vol. 24, no. 3, pp. 271-300, 1997.

[9] S. I. Roumeliotis and J. W. Burdick, "Stochastic cloning: A generalized framework for processing relative state measurements," in Proc. IEEE Int. Conf. Robot. Autom., Washington, DC, 2002, pp. 1788-1795.

[10] B. D. Hoffman, E. T. Baumgartner, T. L. Huntsberger, and P. S. Schenker, "Improved state estimation in challenging terrain," Auton. Robots, vol. 6, no. 2, pp. 113-130, Apr. 1999.
[11] A. I. Mourikis and S. I. Roumeliotis, "On the treatment of relative-pose measurements for mobile robot localization," in Proc. IEEE Int. Conf. Robot. Autom., Orlando, FL, May 15-19, 2006, pp. 2277-2284.

[12] S. I. Roumeliotis (2002, Mar.). A Kalman filter for processing 3-D relative pose measurements. Calif. Inst. Technol., Pasadena, Tech. Rep. [Online]. Available: http://robotics.caltech.edu/ stergios/tech_reports/ relative_3d_kf.pdf

[13] S. Fleischer, "Bounded-error vision-based navigation of autonomous underwater vehicles," Ph.D. dissertation, Stanford Univ., Stanford, CA, 2000.

[14] R. Eustice, H. Singh, and J. Leonard, "Exactly sparse delayed-state filters," in Proc. IEEE Int. Conf. Robot. Autom., Barcelona, Spain, Apr. 2005, pp. 2428-2435.

[15] R. Eustice, O. Pizarro, and H. Singh, "Visually augmented navigation in an unstructured environment using a delayed state history," in Proc. IEEE Int. Conf. Robot. Autom., New Orleans, LA, Apr. 2004, pp. 25-32.

[16] R. Garcia, J. Puig, O. Ridao, and X. Cufi, "Augmented state Kalman filtering for AUV navigation," in Proc. IEEE Int. Conf. Robot. Autom., Washington, DC, May 2002, pp. 4010-4015.

[17] F. Lu and E. Milios, "Globally consistent range scan alignment for environment mapping," Auton. Robots, vol. 4, no. 4, pp. 333-349, 1997.

[18] A. I. Mourikis and S. I. Roumeliotis, "SC-KF mobile robot localization A stochastic cloning-Kalman filter for processing relative-state measurements,” Dept. Comput. Sci. Eng., Univ. Minnesota, Minneapolis, Tech. Rep., Oct. 2006.

[19] E. J. Lefferts, F. L. Markley, and M. D. Shuster, "Kalman filtering for spacecraft attitude estimation," J. Guid. Control Dyn., vol. 5, no. 5, pp. 417-429, Sep./Oct. 1982.

[20] D. Bayard and P. B. Brugarolas, "An estimation algorithm for vision-based exploration of small bodies in space," in Proc. 2005 Amer. Control Conf., Portland, OR, Jun., pp. 4589-4595.

[21] A. I. Mourikis and S. I. Roumeliotis, "A multi-state constraint Kalman filter for vision-aided inertial navigation," in Proc. IEEE Int. Conf. Robot. Autom., Rome, Italy, Apr. 10-14, 2007, pp. 3565-3572.

[22] R. C. Smith, M. Self, and P. Cheeseman, "Estimating uncertain spatial relationships in robotics," Autonomous Robot Vehicles. New York: Springer-Verlag, 1990, pp. 167-193.

[23] M. Montemerlo, "FastSLAM: A factored solution to the simultaneous localization and mapping problem with unknown data association," $\mathrm{Ph} . \mathrm{D}$. dissertation, Robot. Inst., Carnegie Mellon Univ., Pittsburgh, PA, Jul. 2003.

[24] S. Thrun, D. Koller, Z. Ghahramani, and H. Durrant-Whyte, "Simultaneous mapping and localization with sparse extended information filters: Theory and initial results," Sch. Comput. Sci., Carnegie Mellon Univ., Pittsburgh, PA, Tech. Rep., 2002.

[25] P. Newman, J. Leonard, J. D. Tardos, and J. Neira, "Explore and return: Experimental validation of real-time concurrent mapping and localization," in Proc. IEEE Int. Conf. Robot. Autom., Washington, DC, May 11-15, 2002, pp. 1802-1809.

[26] S. J. Julier and J. K. Uhlmann, "Simultaneous localisation and map building using split covariance intersection," in Proc. IEEE/RSJ Int. Conf. Intell. Robots Syst., Maui, HI, Oct. 29-Nov. 32001, pp. 1257-1262.

[27] S. I. Roumeliotis, A. E. Johnson, and J. F. Montgomery, "Augmenting inertial navigation with image-based motion estimation," in 
Proc. IEEE Int. Conf. Robot. Autom., Washington, DC, 2002, pp. 43264333.

[28] P. Pinies and J. D. Tardos, "Fast localization of avalanche victims using sum of Gaussians," in Proc. 2006 IEEE Int. Conf. Robot. Autom., Orlando, FL, May 2006, pp. 3989-3994.

[29] T. Bailey, "Constrained initialisation for bearing-only SLAM," in Proc. IEEE Int. Conf. Robot. Autom., 2003, vol. 2, pp. 1966-1971.

[30] N. M. Kwok and G. Dissanayake, "An efficient multiple hypothesis filter for bearing-only SLAM," in Proc. IEEE/RSJ Int. Conf. Intell. Robots Syst., Sendai, Japan, Oct. 2004, pp. 736-741.

[31] J. Leonard, R. Rikoski, P. Newman, and M. Bosse, "Mapping partially observable features from multiple uncertain vantage points," Int.J. Robot. Res., vol. 21, no. 10/11, pp. 943-975, 2002.

[32] J. Leonard and R. Rikoski, "Incorporation of delayed decision making into stochastic mapping," Exp. Robot. VII, vol. 271, pp. 533-542, 2001.

[33] J. Neira and J. D. Tardos, "Data association in stochastic mapping using the joint compatibility test," IEEE Trans. Robot. Autom., vol. 17, no. 6, pp. 890-897, Dec. 2001.

[34] A. Howard, "Multi-robot mapping using manifold representations," in Proc. 2004 IEEE Int. Conf. Robot. Autom., New Orleans, LA, Apr., pp. 4198-4203.

[35] S. Julier and J. Uhlman, "A non-divergent estimation algorithm in the presence of unknown correlations," in Proc. Amer. Control Conf., vol. 4, 1997, pp. 2369-2373.

[36] S. T. Pfister, S. I. Roumeliotis, and J. W. Burdick, "Weighted line fitting algorithms for mobile robot map building and efficient data representation," in Proc. IEEE Int. Conf. Robot. Autom., 2003, Taipei, Taiwan, R.O.C., pp. 1304-1311.

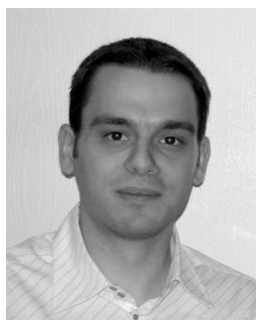

Anastasios I. Mourikis (S'04) received the Diploma of Electrical and Computer Engineering from the University of Patras, Patras, Greece, in 2003. He is currently working toward the Ph.D. degree at the Department of Computer Science and Engineering (CSE), University of Minnesota, Minneapolis.

His current research interests include the areas of localization in single- and multirobot systems, visionaided inertial navigation, simultaneous localization and mapping, and structure from motion.

Mr. Mourikis is the recipient of the 2005 and 2006 Excellence in Research Award Fellowships from the CSE Department, University of Minnesota, and the 2007-2008 Doctoral Dissertation Fellowship from the University of Minnesota.

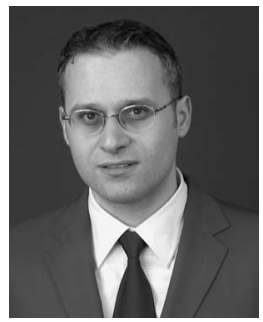

Stergios I. Roumeliotis (M'02) received the Diploma in Electrical Engineering from the National Technical University of Athens, Athens, Greece, in 1995, and the M.S. and Ph.D. degrees in electrical engineering from the University of Southern California, Los Angeles, in 1997 and 2000, respectively.

From 2000 to 2002, he was a Postdoctoral Fellow at California Institute of Technology. Since 2002, he has been an Assistant Professor in the Department of Computer Science and Engineering, University of Minnesota, Minneapolis. His current research interests include inertial navigation of aerial and ground autonomous vehicles, fault detection and identification, sensor networks, distributed estimation under communication and processing constraints, and active sensing for reconfigurable networks of mobile sensors.

Dr. Roumeliotis is the recipient of the National Science Foundation Faculty Early Career Development (NSF CAREER) Award and the McKnight Land-Grant Professorship Award. He is also the corecipient of the NASA Tech Briefs Award, the One NASA Peer Award, and the One NASA Center Best Award. He is currently an Associate Editor for the IEEE TRANSACTIONS ON ROBOTICS.

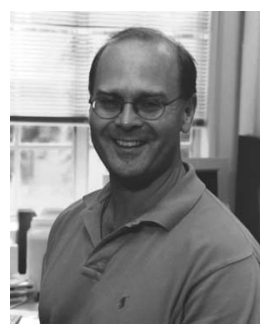

Joel W. Burdick (M'95) received the B.S. degree from Duke University, Durham, NC, in 1981 and the M.S. and Ph.D. degrees from Stanford University, Stanford, CA, all in mechanical engineering in 1982 and 1988, respectively.

Since May 1988, he has been with the Department of Mechanical Engineering, California Institute of Technology, Pasadena. His current research interests include the areas of robotics, mechanical systems, bioengineering, sensor-based robot motion planning, multifingered grasping, coordination of multiple robots, neural prosthetics, and rehabilitation of spinal cord injuries.

Prof. Burdick is the recipient of the National Science Foundation (NSF) Presidential Young Investigator Award, the Office of Naval Research Young Investigator Award, and the Feynman Fellowship. He has also been a finalist of the Best Paper Award for the IEEE International Conference on Robotics and Automation in 1993, 1999, 2000, and 2005. He was appointed an IEEE Robotics Society Distinguished Lecturer in 2003. 